\title{
Long-term outcomes of antipsychotic treatment in patients with first-episode schizophrenia: a systematic review
}

This article was published in the following Dove Press journal:

Neuropsychiatric Disease and Treatment

6 January 2016

Number of times this article has been viewed

\author{
Craig Karson' \\ Ruth A Duffy 2 \\ Anna Eramo ${ }^{3}$ \\ Anna-Greta Nylander ${ }^{4}$ \\ Steve J Offord ${ }^{5}$ \\ 'CNK Consultants, Delray Beach, \\ FL, ${ }^{2}$ Medical Affairs - Neuroscience, \\ Otsuka America Pharmaceutical, \\ Inc., Princeton, NJ, ${ }^{3}$ Medical Affairs \& \\ Phase IV Clinical Affairs, Lundbeck \\ LLC, Deerfield, IL, USA; ${ }^{4}$ Medical \\ Affairs Aripiprazole, $\mathrm{H}$ Lundbeck \\ A/S, Valby, Denmark; ${ }^{5}$ Medical Affairs, \\ Otsuka America Pharmaceutical, Inc., \\ Princeton, NJ, USA
}

Background: Treatment during first-episode psychosis (FEP) or early schizophrenia may affect the rates of relapse and remission, as well as cognitive functioning, over time. Prolonged duration of psychosis is associated with a poor prognosis, but the effects of treatment in patients with FEP or early schizophrenia on the long-term outcomes are not well defined.

Objective: To understand the long-term effects of treatment with antipsychotic agents on remission, relapse, and cognition in patients with FEP or early schizophrenia.

Methods: Using PubMed and Scopus databases, a systematic review was undertaken of articles published between January 1, 2000, and May 20, 2015, that reported randomized and nonrandomized prospective clinical trials on the long-term effects of oral or long-acting injectable antipsychotics on measures of relapse, remission, or cognition in patients with FEP or early schizophrenia. For comparative purposes, trials reporting the effects of later intervention with antipsychotics in patients with longer disease history were also evaluated. Titles, abstracts, and full-text articles were independently screened for eligibility by all the authors based on the predefined criteria.

Results: Nineteen studies met inclusion criteria: 13 reported long-term outcomes of relapse, remission, or cognition following antipsychotic treatment in patients with FEP and six reported on patients with a longer disease history. Antipsychotic treatment in patients with FEP produced high rates of remission in the year following treatment initiation, and untreated FEP reduced the odds of later achieving remission. Maintenance therapy was more effective than treatment discontinuation or intermittent/guided discontinuation in preventing relapse. Initiating antipsychotic treatment in patients with FEP also produced sustained cognitive improvement for up to 2 years. Antipsychotic therapy also reduced the risk or rate of relapse in patients with a longer disease history, with outcomes in one study favoring a long-acting injectable formulation over an oral antipsychotic.

Conclusion: Treatment of patients with FEP is associated with benefits in the long-term outcomes of remission, relapse, and cognition. More long-term studies of treatment in patients with FEP are needed to confirm these findings.

Keywords: cognition, first-episode psychosis, long-term outcome, relapse, remission, schizophrenia

\section{Introduction}

Schizophrenia is a chronic illness associated with periods of relapse and remission and poor long-term outcomes. ${ }^{1}$ The period surrounding the first episode of psychosis represents a critical juncture in the natural course of the disease, particularly in young patients for whom symptoms and impaired functioning may be less well established. ${ }^{2,3}$ Untreated psychosis may have untoward effects on neurobiologic function, and lack of intervention in first-episode psychosis (FEP) or early schizophrenia may lead
Correspondence: Craig Karson CNK Consultants, 220 Macfarlane Drive \#405, Delray Beach, FL 33483, USA

Tel + I 617388423 |

Fax +I 56I 2788288

Email brainiacle520@gmail.com
Neuropsychiatric Disease and Treatment 2016:12 57-67 
to prolonged times to remission later in the course of the disease, as well as reduced recovery and potential treatment resistance. ${ }^{2}$ At least $50 \%$ of patients with schizophrenia receiving either intermittent or continuous antipsychotic treatment experience occasions of unremitting hallucinations, delusions, disorganized thinking, and negative symptoms $>10$ years after disease onset, and this can disrupt the development of interpersonal and occupational skills., ${ }^{2,4}$

A recently published systematic review and meta-analysis demonstrated that a long duration of untreated psychosis has small but statistically significant correlations with poor overall symptomatic outcome as well as more severe positive and negative symptoms, failure to achieve remission, impaired social functioning, and decreased global outcome (eg, combined clinical/social outcome or outcome on Global Assessment of Functioning scale). ${ }^{5}$ These results suggest that intervention in FEP or early schizophrenia may benefit long-term outcomes.

A number of recent systematic reviews and meta-analyses have evaluated the efficacy of antipsychotics in relapse prevention and in FEP or early schizophrenia. ${ }^{6-10}$ However, none have addressed the long-term impact of intervention in FEP or early schizophrenia on the key outcomes of remission, relapse, or cognition. Ventura et $\mathrm{al}^{11}$ demonstrated that the Wechsler Adult Intelligence Scale comprehension score, a measure of cognition, was a good predictor of functional outcome at 6 months following FEP in schizophrenia. This is consistent with prior studies associating cognition with functional outcome. ${ }^{12-14} \mathrm{We}$ chose to focus on cognition because it is a pervasive aspect of schizophrenia; cognitive deficits may contribute to poor functional and social outcomes and diminished quality of life. ${ }^{15}$ Cognitive impairment may worsen with disease duration but may be amenable to long-term improvement accompanying control of psychopathology. Thus, it was of interest to assess whether evidence was available showing that starting antipsychotic treatment early in the disease course could improve or stabilize cognitive functioning. The objective of this systematic review was to evaluate the effect of treatment with oral and long-acting injectable (LAI) antipsychotics on long-term outcomes in patients with FEP or early schizophrenia. For comparing and contrasting the differences when treatment is started later in the course of the disease, studies assessing long-term outcomes when treating patients with a longer disease history were also examined.

\section{Materials and methods}

\section{Search methodology}

Systematic searches of the PubMed and Scopus databases were performed on May 20, 2014, and again on May 20, 2015, using the following search string: (schizophrenia OR psychosis) AND antipsychotic AND ("first episode" OR "first-episode" OR "new onset" OR "early schizophrenia" OR “early intervention” OR “long-term” OR “chronic schizophrenia” OR “chronic unstable schizophrenia”) AND (cognition OR cognitive OR relapse OR remission OR recovery). The searches were limited to English language articles and the title/abstract (PubMed) or abstract (Scopus) fields; for Scopus, the source type was limited to journals. To avoid double counting of studies, meta-analyses identified during the search were not included in the analysis. All publications that did not include the search terms in the title or abstract were excluded from the review.

\section{Study selection}

All authors independently evaluated the articles for eligibility, beginning with the titles and proceeding sequentially to abstracts and full text. Studies of interest reported data from prospective randomized and nonrandomized clinical trials, evaluating the effect of an oral or LAI antipsychotic in patients with FEP or early schizophrenia with respect to longterm relapse, remission, and/or cognition. The references cited in studies that met inclusion/exclusion criteria were also reviewed for additional potentially eligible articles. For comparative purposes, studies of patients with longer disease history that met all other inclusion/exclusion criteria were included in the review.

By mutual author agreement, studies focused on study design, health economics, and outcomes research were excluded. Studies reporting cross-sectional, retrospective, or secondary analysis were also excluded. All publications that did not include measures of remission, relapse, or cognition as primary end points or were within primary publications were excluded. Other exclusion criteria included studies with a low number of patients (ie, $\mathrm{n}<50$ ), duration $\leq 12$ weeks, publication date before 2000, drug no longer used as an antipsychotic, polypharmacy or augmentation therapy, neural or biochemical outcomes, elderly patients, comorbid substance abuse, depression, and multiple treatment indications without separation of data reporting. The authors agreed that studies involving $<50$ patients would yield less robust data and conclusions than those with at least 50 patients. The year 2000 was chosen as a cutoff to keep the review timely because many treatment advances have been implemented since then, which have in turn affected the clinical practice. Disagreements among the authors were resolved by group discussion. Majority rule was applied to conflicting opinions to determine the eligibility. Statistical analysis of the data was not performed. 


\section{Results}

\section{Search results: study characteristics}

Study identification, screening, and eligibility are summarized in Figure 1. Database searching yielded 785 unique references; 511 references were excluded after title screening and 179 were excluded after abstract screening. The remaining 95 articles were subjected to full-text review, during which another seven potential articles were identified and subsequently reviewed for inclusion. Of the 102 articles reviewed in full, 83 were excluded, leaving 19 articles: 13 included analysis of long-term outcomes of remission or relapse $^{16-20}$ (Table 1) or cognition ${ }^{21-27}$ (Table 2) in patients with FEP and six reported on patients with a longer disease history ${ }^{28-33}$ (Table 3).

Among the studies including patients defined as having FEP, the patient populations varied broadly with respect to disease duration and prior antipsychotic exposure. Disease duration ranged from 1 month to 5 years. One study did not report disease duration or did not include adequate data to determine the disease duration. ${ }^{16}$ Duration of prior antipsychotic exposure ranged from none (treatment naïve) to a median of 21 months; when excluding the study of patients with remitted FEP, ${ }^{16}$ the maximum duration of exposure was $\leq 16$ weeks. There were two studies that did not report duration of prior antipsychotic exposure, ${ }^{17,20}$ however, in one of the studies, the authors noted that $54 \%$ of the patients were treatment naïve at baseline. ${ }^{17}$ Of note, in one study, patients were not required to have FEP but had to be in the early phase of schizophrenia, defined as $\leq 5$ years since their first exposure to neuroleptic treatment; the mean disease duration ranged from 2.45 years to 2.79 years among treatment groups. ${ }^{27}$ This particular study was included in the analysis of early schizophrenia because the authors specifically identified the patients as "in the early phase of schizophrenia," although it is possible that the patient population overlapped with those in the comparative, later phase studies.

For comparative purposes, there were six studies of patients with schizophrenia or schizoaffective disorder and a longer disease history that met the inclusion criteria (Table 3). ${ }^{28-33}$ Disease durations ranged from 10 years to 17 years. ${ }^{28-30,32,33}$ One study did not report disease duration

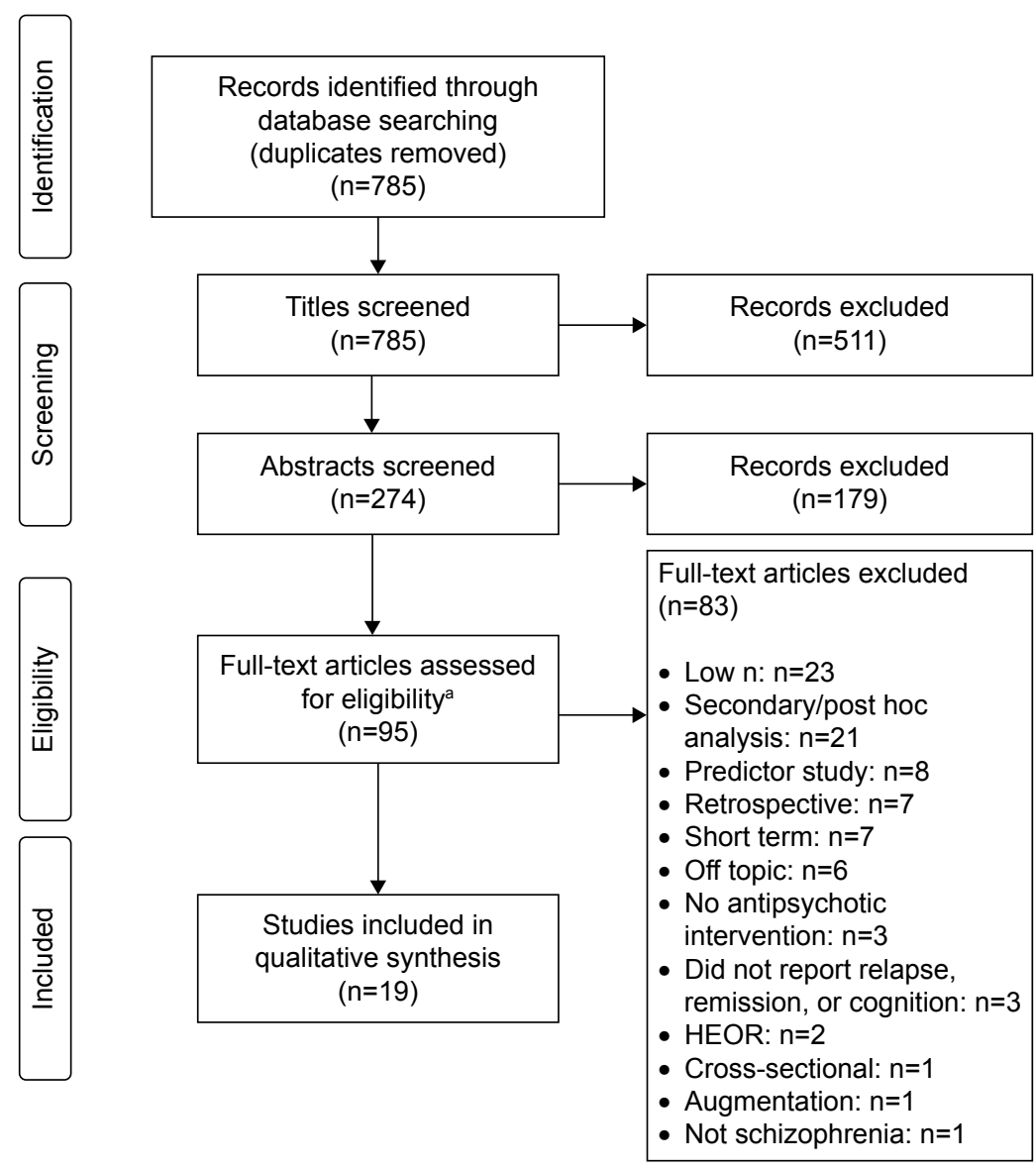

Figure I Flow diagram of article selection.

Note: additional full-text articles $(n=7)$ were identified from the references cited in studies that met inclusion/exclusion criteria and were also assessed for eligibility. Abbreviation: HEOR, health economics and outcomes research. 


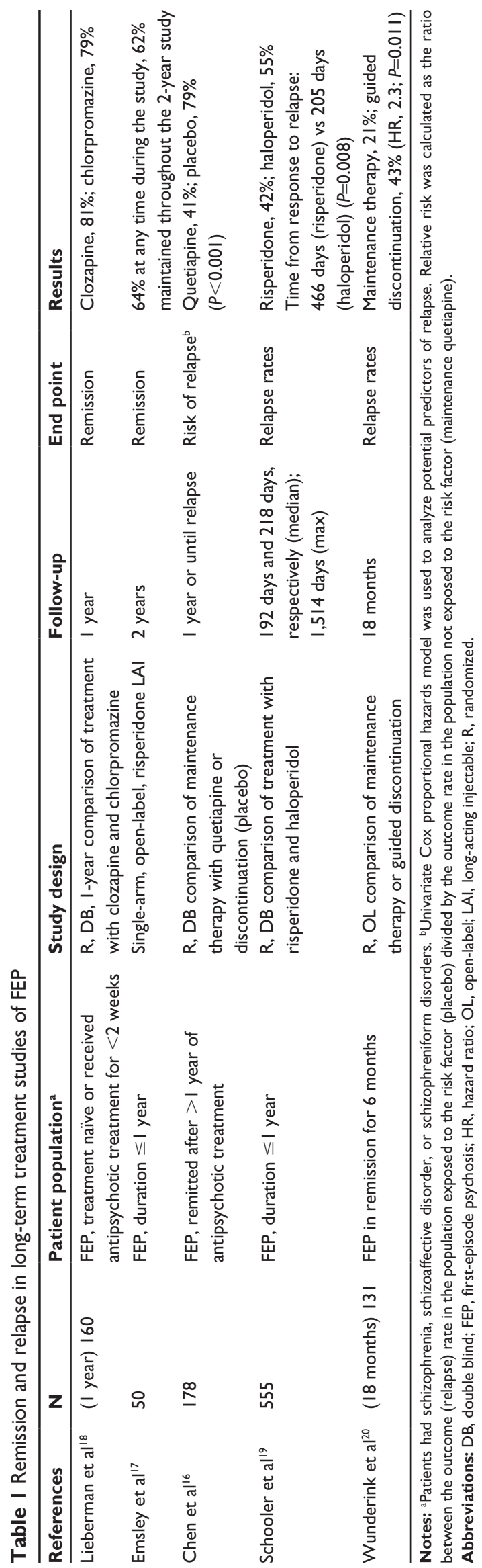

and did not include adequate data to determine the disease duration; however, patients were required to have diagnoses of schizophrenia $\geq 2$ years prior to study entry as part of the study's inclusion criteria. ${ }^{31}$ Most of the studies in patients with longer disease history included clinically stable patients with residual symptoms; ${ }^{28,29,31-33}$ one study included patients who were currently experiencing a relapse.$^{30}$ Relapse (risk, time to, or rate of) was reported as a primary outcome in five studies, ${ }^{28-32}$ and cognitive function was reported as a primary outcome in one study. ${ }^{33}$ Definitions of relapse were based on criteria that included psychiatric hospitalization, increased level of psychiatric care, predefined absolute or percentage increase on the Positive and Negative Syndrome Scale (PANSS) total score, predefined increases on individual PANSS items, predefined decreases on the Brief Psychiatric Rating Scale (BPRS), Clinical Global Impression (CGI) Improvement score of $\geq 5$ (minimally worse), deliberate selfinjury, clinically significant suicidal or homicidal ideation, violent behavior (ie, injuring someone or significant property damage), or an adverse event of worsening schizophrenia.

\section{Remission and relapse}

Remission as an outcome was examined in two FEP studies that met the inclusion criteria for this review (Table 1): a randomized, double-blind study that evaluated the onset and duration of remission in 160 treatment naïve patients with FEP after 1 year of treatment with clozapine or chlorpromazine ${ }^{18}$ and a single-arm, 2-year, open-label study that evaluated remission, time to remission, and maintenance of remission in 50 patients (54\% treatment naïve) treated with risperidone LAI. ${ }^{17}$ Patients in the double-blind study ${ }^{18}$ had scored $\geq 4$ (moderate severity) on one or more of the five psychosis items on the BPRS at the time of screening. The primary end point, time to first remission, was defined as meeting the following criteria: $\geq 50 \%$ reduction from baseline in the BPRS total score, no score $>3$ (mild) on the five BPRS psychosis items, and a CGI-severity score $\leq 3$ (mildly ill); in this study, remission did not require that these criteria be met for a specified minimal duration. A high degree of treatment responsiveness was reported for both drugs at study end point, with no differences in the rates of achieving remission before discontinuing or completing the 52-week study (clozapine, $81 \%$; chlorpromazine, $79 \%$ ). However, time to remission was more rapid (hazard ratio [HR], 1.69; 95\% confidence interval [CI], 1.18-2.44; $P=0.005)$ and time spent in remission was longer (odds ratio [OR], 1.73; 95\% CI, 1.20-2.50; $P=0.003$ ) with clozapine compared with chlorpromazine treatment. The median time to first remission was 8 weeks with clozapine 


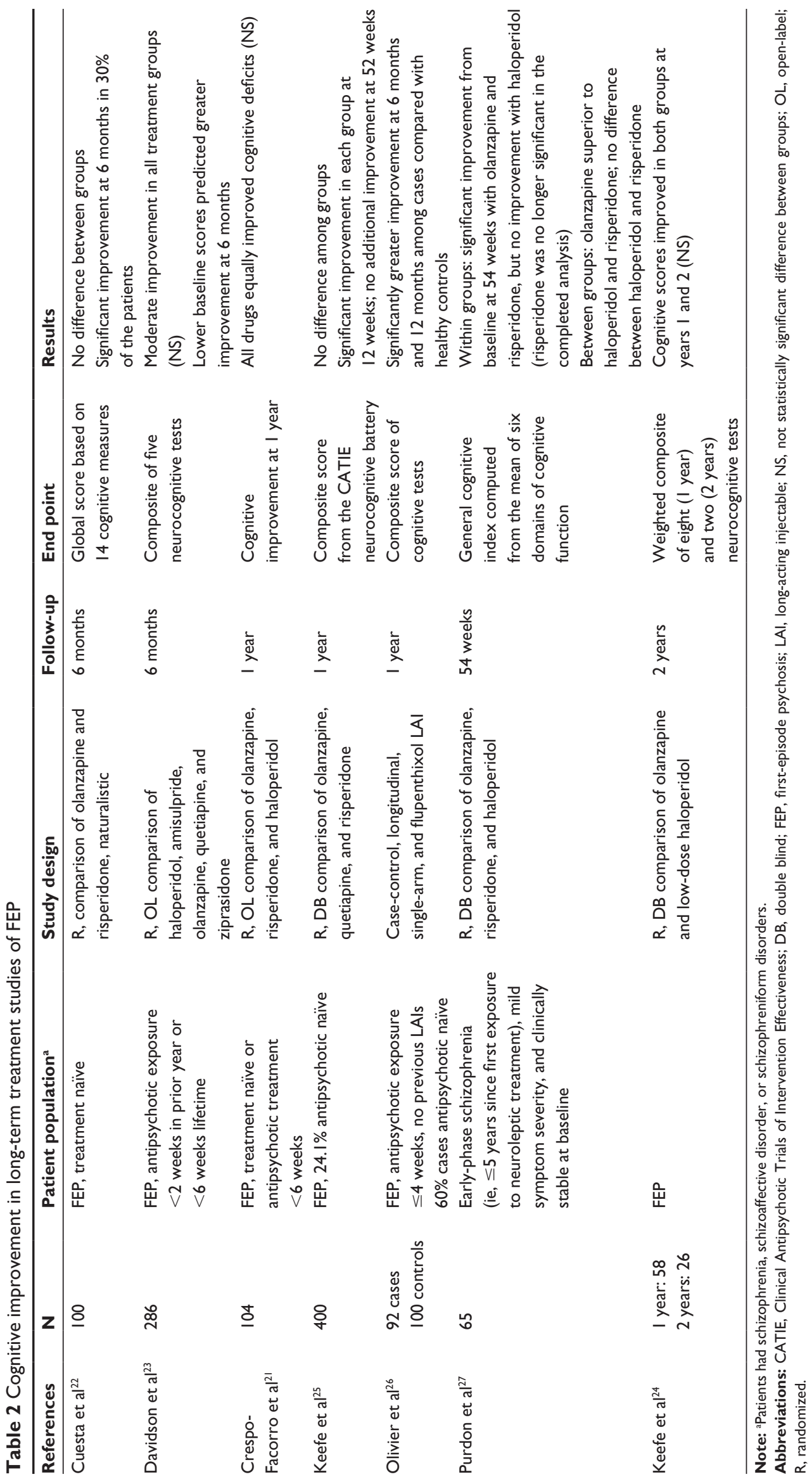




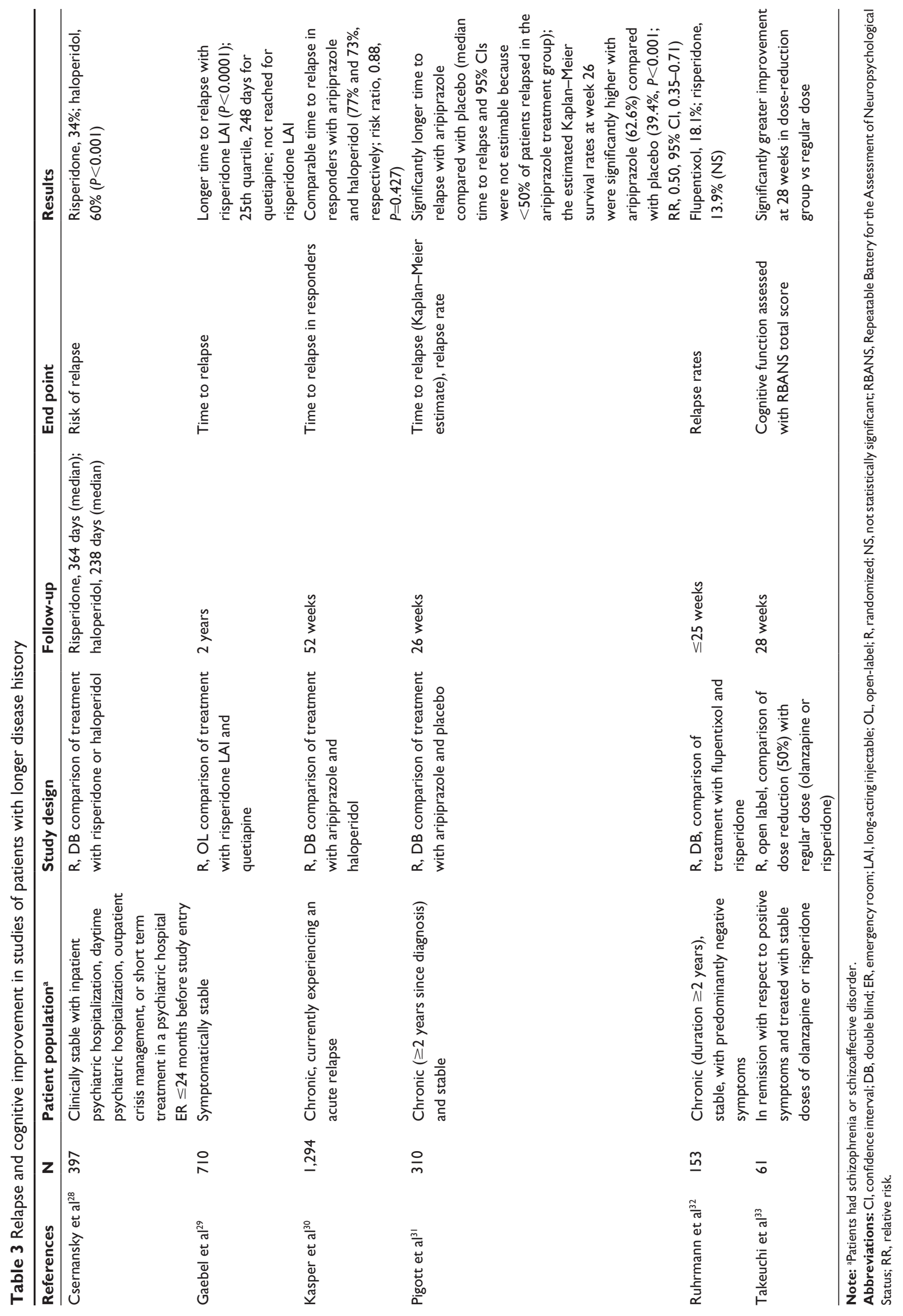


versus 12 weeks with chlorpromazine, $\chi^{2}(1)=5.56, P=0.02$. Logistic regression modeling demonstrated that for every year of untreated psychosis, the odds of achieving remission decreased by $15 \%$ (OR, $0.85 ; 95 \%$ CI, $0.75-0.95 ; P=0.006){ }^{18}$ The open-label risperidone LAI study used the Remission in Schizophrenia Working Group criteria of remission that require maintaining mild or lower levels $(\leq 3)$ on eight key PANSS items for $\geq 6$ months. ${ }^{17}$ The median time to remission was 301 days; $64 \%$ of patients achieved remission during the study and $62 \%$ maintained remission throughout the study.

Data on relapse prevention in patients with FEP were also scarce. Three articles evaluating relapse met the inclusion criteria for this review: two studies examined relapse rates and one study evaluated the risk of relapse and relapse rate (Table 1). ${ }^{16,19,20}$ Definitions of relapse were based on the criteria that included meeting predefined scores on PANSS items; ${ }^{20}$ meeting predefined scores on PANSS items and CGI-severity and improvement scores ${ }^{16}$ or meeting predefined increases on PANSS total scores, CGI changes of "much worse" or "very much worse," and adverse events of deliberate self-injury, clinically significant suicidal or homicidal ideation, or violent behavior (ie, injuring someone or significant property damage). ${ }^{19}$

The time course of relapse was examined in a study of 555 patients with FEP who were randomized to receive treatment with haloperidol or risperidone and were followed for up to 4 years. ${ }^{19}$ In this cohort, the median time to relapse was 205 days in patients treated with haloperidol and 466 days in patients treated with risperidone $(P=0.008)$; the overall relapse rates were $55 \%$ and $42 \%$, respectively, in 400 of the patients who initially achieved clinical improvement.

Maintenance treatment in patients with FEP has also been compared with planned discontinuation. Maintenance treatment with antipsychotic agents for up to 1 year prevents relapse in patients with stable, remitted FEP and is more effective than the strategy of treatment discontinuation (Table 1). In the study reported by Chen et al, 178 patients with FEP who had completed $\geq 1$ year of antipsychotic drug treatment with no relapses were randomized to 1 year of maintenance therapy with quetiapine or had a supervised treatment discontinuation (matched placebo). ${ }^{16}$ At baseline, patients had no psychotic symptoms for $\geq 8$ weeks and had been treated with antipsychotics for a mean of 618 days. Univariate Cox proportional hazards model was used to analyze the potential predictors of relapse, and relative risk (RR) was calculated as the ratio between the outcome (relapse) rate in the population exposed to the risk factor (placebo) divided by the outcome rate in the population not exposed to the risk factor (maintenance quetiapine). Risk of relapse at 12 months was $41 \%$ for patients receiving maintenance therapy $(95 \% \mathrm{CI}, 29 \%-53 \%)$ and $79 \%$ for patients undergoing treatment discontinuation (95\% CI, 68\%-90\%; $P<0.001)$.

In a study reported by Wunderink et al, 131 stabilized patients with FEP with antipsychotic therapy for 6 months were randomly assigned to receive maintenance therapy or to undergo guided discontinuation (symptom-guided tapering) ${ }^{20}$ After 18 months follow-up, the relapse rates were $21 \%$ for patients receiving maintenance therapy and $43 \%$ among patients assigned to guided discontinuation (HR, 2.3; $P=0.011$ ).

Five studies met our search criteria for studies that reported relapse and/or remission in patients with chronic schizophrenia: one placebo-controlled trial, three studies comparing second-generation antipsychotic (SGA) and first-generation antipsychotic (FGA), and one study comparing an LAI with an oral SGA (Table 3). ${ }^{28-32}$ A 26-week, randomized, controlled trial in patients with chronic ( $\geq 2$ years), stable (no significant improvement or worsening $\leq 3$ months prior to study entry) schizophrenia reported significantly higher estimated Kaplan-Meier survival rates at week 26 with oral aripiprazole compared with placebo (relapse rate, $62.6 \%$ vs $39.4 \%$, respectively; $P<0.001$; RR, $0.50,95 \% \mathrm{CI}, 0.35-0.71)$. The median time to relapse and $95 \%$ CIs were not estimable because $<50 \%$ of patients in the aripiprazole treatment group relapsed.$^{31}$ In one study of patients with chronic schizophrenia (mean age at onset, $\sim 25$ years; mean age at study entry, $\sim 37$ years) who were experiencing an acute relapse, high and comparable response rates were observed with the SGA aripiprazole $(72 \%)$ and the FGA haloperidol $(69 \%)(P=0.362) .{ }^{30}$ The relapse rates were also comparable between the SGA risperidone (13.9\%) and FGA flupentixol (18.1\%) in a randomized, double-blind study in patients with chronic schizophrenia (mean time since diagnosis, $\sim 11$ years) with predominantly negative symptoms.$^{32}$ In another study, in which patients had a mean illness duration of $>10$ years, the Kaplan-Meier estimated risk of relapse at 1 year was significantly lower with risperidone $(34 \%)$ compared with haloperidol $(60 \%)(P<0.001) .{ }^{28}$ Switching to a second-generation LAI formulation was shown to provide further benefit in a 2-year, randomized clinical trial in patients with a mean ( \pm standard deviation) time since diagnosis of $9.9( \pm 9.9)$ to $10.0( \pm 10.1)$ years. In that study, the RR of relapse in patients who switched to risperidone LAI was less than half for those who switched 
to oral quetiapine from other oral antipsychotics (HR, 0.46; $97 \%$ CI, 0.32-0.67). ${ }^{29}$

In summary, two studies reported remission as a primary outcome in patients with FEP: a double-blind, randomized, 1-year trial in which a similar proportion of patients achieved remission but achieved it earlier and sustained it longer with clozapine versus chlorpromazine ${ }^{18}$ and an open-label, 2-year, single-arm study with risperidone LAI in which almost two-thirds of patients achieved remission and remained in remission through the end of the study..$^{17}$ Another three studies $^{16,19,20}$ assessed relapse rate, timing of relapse, or the risk of relapse in patients with FEP. In one of these studies, relapse rates were lower and median time to relapse was longer for risperidone versus haloperidol. ${ }^{19}$ In two studies assessing discontinuation versus maintenance treatment in patients in remission after FEP, maintenance treatment halved the risk of relapse versus placebo (discontinuation) ${ }^{16}$ and halved relapse rates versus guided discontinuation. ${ }^{20}$

\section{Cognition}

Neurocognitive deficits are an established clinical dimension associated with both early and chronic schizophrenia. ${ }^{15}$ Treatment with atypical antipsychotic agents has been shown to reduce long-standing cognitive deficits in some patients with chronic, treatment-resistant schizophrenia; however, improvements are modest, although statistically significant. ${ }^{34,35}$

Durable cognitive benefits from atypical antipsychotics have been observed in patients with FEP following treatment ranging from 6 months to 2 years (Table 2). Cognition was examined as a primary outcome in seven articles that met the inclusion criteria. ${ }^{21,23-27}$ Primary end points included changes in individual tests or composite scores derived from cognitive tests across multiple domains (eg, verbal memory, visual memory, executive functioning, working memory, speed of processing, and attention). All but one of the studies included one or more motor speed/coordination tests in the composite score. ${ }^{23-25,27}$

Four of the seven studies that examined cognitive functioning included comparisons of FGAs with SGAs, ${ }^{21,23,24,27}$ and all but one of the studies ${ }^{27}$ reported comparable improvements in cognitive assessments between the FGA and SGA treatment groups. In three of the four studies that assessed multiple SGAs, comparisons between SGAs revealed no within-group differences. ${ }^{21,23,25}$ The one study that found between-group differences in cognitive functioning with SGAs versus FGAs was conducted in patients with early phase schizophrenia, where significant improvements from baseline at 54 weeks on a composite score of cognitive functioning were observed with olanzapine and risperidone but not with haloperidol. ${ }^{27}$ However, in alternate analyses using a repeated-measures model, results for risperidone were no longer statistically significant in patients who completed the study. Between-group comparisons revealed significantly greater improvement in the composite score with olanzapine compared with haloperidol and risperidone, but no significant difference between haloperidol and risperidone. In general, poor baseline performance on neuropsychologic tests predicted greater cognitive improvement. ${ }^{22,23}$

There was one study of patients with chronic schizophrenia that included measures of cognition and that met the inclusion/exclusion criteria. This open-label, 28-week, randomized, controlled pilot study examined the impact of dose reduction on cognitive function in stable patients with schizophrenia who were treated for $\geq 3$ months with oral risperidone $(>2 \mathrm{mg} / \mathrm{d})$ or oral olanzapine $(>5 \mathrm{mg} / \mathrm{d}){ }^{33}$ Patients were randomized to $50 \%$ dose reduction (or one-third dose reduction, if needed to remain within the lower limit of the recommended dose range) or treatment at their usual dose (standard treatment) and assessed for cognitive function with the Repeatable Battery for the Assessment of Neuropsychological Status (RBANS) total score. Compared with the standard treatment, the reduced dose significantly improved patients' cognitive function at 28 weeks, and exploratory analyses revealed that the difference between the two groups in the RBANS total score was driven by improvements in immediate memory and language scores.

\section{Discussion}

Treatment of early schizophrenia results in favorable longterm outcomes. Treatment with atypical antipsychotics may result in a faster and more sustained remission compared with FGAs. ${ }^{18}$ In general, treatment in patients with FEP has a markedly positive effect on the likelihood of remission ${ }^{18}$ and risk ${ }^{16}$ or rate ${ }^{20}$ of relapse. Although the effect is associated with both oral and LAI antipsychotics, one study reported a rate of remission sustained for $\geq 6$ months with LAIs that compared favorably relative to that reported in FEP treated with oral antipsychotics. ${ }^{17}$ Patients with a longer duration of schizophrenia (ie, chronic schizophrenia) also demonstrated reduced risk of relapse with antipsychotic therapy versus placebo in one study included in our review, ${ }^{31}$ and in another study, a second-generation LAI conferred greater protection from relapse than an oral FGA. ${ }^{29}$ Regardless, given the reduced likelihood of remission with longer duration of untreated psychosis, ${ }^{18}$ earlier 
intervention may be best for achieving treatment goals over the long term.

Long-term relapse prevention is an important goal of schizophrenia treatment. ${ }^{7}$ Given the chronic course of schizophrenia, the typical early age at onset, and the adverse events associated with antipsychotic treatment, ${ }^{36}$ it would seem rational to attempt trials of drug discontinuation over the course of the disease. However, the results of two studies of treatment discontinuation or intermittent/guided discontinuation identified in this systematic review demonstrate that uninterrupted maintenance therapy for up to 2 years is a significantly more effective option for preventing relapses in patients with FEP. ${ }^{16,20}$ These findings are in concordance with the observation that, in patients with recent-onset schizophrenia who underwent supervised discontinuation of antipsychotic treatment, exacerbation or relapse of schizophrenia occurs in $78 \%$ of patients in the first year and $96 \%$ of patients by the second year. ${ }^{37}$ Nonadherence with antipsychotic medication may also contribute to poor long-term outcome in patients with FEP. ${ }^{38}$ The results from a nationwide cohort study in Finland show poor antipsychotic treatment adherence in FEP and a significantly lower risk of rehospitalization associated with LAI versus oral formulations. ${ }^{38}$ Early treatment and patient discharge with LAI antipsychotics versus oral formulations may improve outcomes in patients with FEP.

Improvements in cognitive impairment are another key target in the treatment of schizophrenia. ${ }^{36}$ There is substantial evidence that impaired cognition is present in many patients with FEP. ${ }^{39-41}$ Treatment of FEP does confer cognitive benefits, with relatively modest effects observed from 6 months ${ }^{23}$ to 2 years. ${ }^{24}$ Practice effect may account for some of the observed gains. Crespo-Facorro et al reported that most changes in cognitive score were similar to those attributed to practice effects observed in healthy controls, but in some cognitive variables, the performance gains in patients exceeded those in controls. ${ }^{21}$ In a study that included patient-matched healthy volunteers, improvements observed in controls were of a smaller magnitude than those in the patients. ${ }^{26}$ Cuesta et al, who did not include a control group in the study design, noted that cognitive improvements were observed not only in tests prone to familiarity and procedural learning but also across the spectrum. ${ }^{22}$ Practice effect cannot be completely excluded from interpretation of neurocognitive tests; however, it is reasonable to conclude that at least part of the reported cognitive improvement is related to the antipsychotic intervention, especially in studies with long elapsed time between testing. ${ }^{23}$
Although antipsychotics improve patients' neurocognitive function, the deficit is still notable compared with healthy controls. ${ }^{21,24,26}$ The magnitude of improvement in neurocognitive test performance appears to be greater in antipsychotic naïve patients relative to those exposed to antipsychotics prior to baseline assessment ${ }^{24,25}$ and also in patients with more severe baseline cognitive deficits compared with those having less severe dysfunction. ${ }^{22,23}$ Patients previously treated with antipsychotics may have a reduced neurocognitive response to further treatment as suggested by results demonstrating that initial cognitive improvements at early time points are not followed by additional gains at later time points. ${ }^{25,26}$ Although such a possibility is not addressed by the studies in our review, identifying patients who may develop schizophrenia through genotyping ${ }^{42,43}$ or other means ${ }^{44}$ may provide an opportunity to intervene before cognitive deficits become pronounced or irreversible.

How long-term cognitive improvements associated with FGA or SGA antipsychotics may differ between patients with recent-onset schizophrenia and patients with a longer course of disease remains unknown, although there is evidence that cognitive performance of patients with schizophrenia may be negatively affected by high dosing of antipsychotics. ${ }^{45}$ It is noteworthy that cognitive function, processing speed in particular, improved in patients with chronic schizophrenia when their doses of atypical antipsychotics were reduced to the lower limit of currently recommended doses. ${ }^{33}$ However, this finding requires confirmation in a larger and longer double-blind study, and its results preclude generalization, especially to FEP, because of the particular characteristics of the study population. The study population comprised clinically stable patients with a long history of the illness and without significant positive symptoms; such patients may be at a relatively low risk of relapse when treated with reduced doses of antipsychotics.

The objective of our systematic review, to determine the impact of early intervention (ie, in FEP or early schizophrenia) on long-term outcomes of remission, relapse, and cognitive functioning, was clearly defined and entailed careful selection of manuscripts that met the objective. However, the meticulous criteria also narrowed our search to a selected number of journal articles that may not represent all studies in patients with FEP and thus may limit the generalizability of our findings. The small number of articles meeting our criteria emphasizes the need for additional rigorous studies designed to assess long-term outcomes in patients with FEP in schizophrenia. We included studies for which the primary outcome was assessed after 6 months, although a longer time 
frame would likely provide a stronger indication of long-term outcomes. Moreover, within the FEP studies, the specific patient inclusion criteria were highly variable, indicating a lack of uniformity in the terminology that could also affect the generalizability of the conclusions. It should also be noted that, overall, among the studies, there was a high degree of heterogeneity in the patients' characteristics, and treatment effects may have been influenced by factors such as the disease severity or genetic variation. ${ }^{46}$ To provide context for the results in patients with FEP, we assessed similar studies in patients with chronic symptomatic schizophrenia. It is notable that there were no studies identified in our search that directly compared outcomes in patients with FEP and those with a longer history of symptomatic schizophrenia; such studies would provide the opportunity to draw conclusions about the potential differences between early and later treatments.

\section{Conclusion}

Antipsychotic treatment of patients with FEP appears to confer long-term benefit with regard to maintenance of remission, prevention of relapse, and improvement in cognitive deficits. Delaying treatment may reduce the odds of achieving remission. Primary analyses of long-term outcomes with treatment of FEP are needed to inform clinical decision making during this important period in the course of schizophrenia.

\section{Acknowledgments}

This research was supported by Otsuka America Pharmaceutical, Inc., and H Lundbeck A/S. Otsuka America Pharmaceutical, Inc., and H Lundbeck A/S also funded editorial support for the preparation of this manuscript, provided by $\mathrm{C} 4 \mathrm{Med}$ Solutions LLC (Yardley, PA), a CHC Group company.

\section{Disclosure}

Craig Karson is a paid consultant for Otsuka America Pharmaceutical, Inc. Ruth A. Duffy and Steve J Offord are employees of Otsuka America Pharmaceutical, Inc. AnnaGreta Nylander is an employee of H Lundbeck A/S. Anna Eramo is an employee of Lundbeck LLC. The authors report no other conflicts of interest in this work.

\section{References}

1. Robinson D, Woerner MG, Alvir JM, et al. Predictors of relapse following response from a first episode of schizophrenia or schizoaffective disorder. Arch Gen Psychiatry. 1999;56(3):241-247.

2. Sheitman BB, Lieberman JA. The natural history and pathophysiology of treatment resistant schizophrenia. J Psychiatr Res. 1998;32(3-4): $143-150$.
3. Lieberman JA, Sheitman B, Chakos M, et al. The development of treatment resistance in patients with schizophrenia: a clinical and pathophysiologic perspective. J Clin Psychopharmacol. 1998;18(2 suppl 1): 20S-24S.

4. Hegelstad WT, Larsen TK, Auestad B, et al. Long-term follow-up of the TIPS early detection in psychosis study: effects on 10-year outcome. Am J Psychiatry. 2012;169(4):374-380.

5. Penttila M, Jaaskelainen E, Hirvonen N, et al. Duration of untreated psychosis as predictor of long-term outcome in schizophrenia: systematic review and meta-analysis. Br J Psychiatry. 2014;205(2):88-94.

6. Alvarez-Jimenez M, Parker AG, Hetrick SE, et al. Preventing the second episode: a systematic review and meta-analysis of psychosocial and pharmacological trials in first-episode psychosis. Schizophr Bull. 2011;37(3):619-630.

7. Emsley R, Chiliza B, Asmal L, et al. The nature of relapse in schizophrenia. BMC Psychiatry. 2013;13:50.

8. Leucht S, Tardy M, Komossa K, et al. Antipsychotic drugs versus placebo for relapse prevention in schizophrenia: a systematic review and meta-analysis. Lancet. 2012;379(9831):2063-2071.

9. Marshall M, Rathbone J. Early intervention for psychosis. Cochrane Database Syst Rev. 2011;6:CD004718.

10. Zipursky RB, Menezes NM, Streiner DL. Risk of symptom recurrence with medication discontinuation in first-episode psychosis: a systematic review. Schizophr Res. 2014;152(2-3):408-414.

11. Ventura J, Subotnik KL, Guzik LH, et al. Remission and recovery during the first outpatient year of the early course of schizophrenia. Schizophr Res. 2011;132(1):18-23.

12. Green MF, Kern RS, Braff DL, et al. Neurocognitive deficits and functional outcome in schizophrenia: are we measuring the "right stuff"? Schizophr Bull. 2000;26(1):119-136.

13. Leeson VC, Barnes TR, Hutton SB, et al. IQ as a predictor of functional outcome in schizophrenia: a longitudinal, four-year study of first-episode psychosis. Schizophr Res. 2009;107(1):55-60.

14. Robinson DG, Woerner MG, McMeniman M, et al. Symptomatic and functional recovery from a first episode of schizophrenia or schizoaffective disorder. Am J Psychiatry. 2004;161(3):473-479.

15. Keefe SE, Harvey PD. Cognitive impairment in schizophrenia. In: Geyer MA, Gross G, editors. Novel Antischizophrenia Treatments, Handbook of Experimental Pharmacology 2013. Heidelberg: Springer Verlag; 2012:11-39.

16. Chen EY, Hui CL, Lam MM, et al. Maintenance treatment with quetiapine versus discontinuation after one year of treatment in patients with remitted first episode psychosis: randomised controlled trial. BMJ. 2010;341:c4024.

17. Emsley R, Oosthuizen P, Koen L, et al. Remission in patients with first-episode schizophrenia receiving assured antipsychotic medication: a study with risperidone long-acting injection. Int Clin Psychopharmacol. 2008;23(6):325-331.

18. Lieberman JA, Phillips M, Gu H, et al. Atypical and conventional antipsychotic drugs in treatment-naive first-episode schizophrenia: a 52-week randomized trial of clozapine vs chlorpromazine. Neuropsychopharmacology. 2003;28(5):995-1003.

19. Schooler N, Rabinowitz J, Davidson M, et al. Risperidone and haloperidol in first-episode psychosis: a long-term randomized trial. Am J Psychiatry. 2005;162(5):947-953.

20. Wunderink L, Nienhuis FJ, Sytema S, et al. Guided discontinuation versus maintenance treatment in remitted first-episode psychosis: relapse rates and functional outcome. $J$ Clin Psychiatry. 2007;68(5): 654-661.

21. Crespo-Facorro B, Rodriguez-Sanchez JM, Perez-Iglesias R, et al. Neurocognitive effectiveness of haloperidol, risperidone, and olanzapine in first-episode psychosis: a randomized, controlled 1-year follow-up comparison. J Clin Psychiatry. 2009;70(5):717-729.

22. Cuesta MJ, Jalon EG, Campos MS, et al. Cognitive effectiveness of olanzapine and risperidone in first-episode psychosis. Br J Psychiatry. 2009;194(5):439-445. 
23. Davidson M, Galderisi S, Weiser M, et al. Cognitive effects of antipsychotic drugs in first-episode schizophrenia and schizophreniform disorder: a randomized, open-label clinical trial (EUFEST). Am J Psychiatry. 2009;166(6):675-682.

24. Keefe RS, Seidman LJ, Christensen BK, et al. Long-term neurocognitive effects of olanzapine or low-dose haloperidol in first-episode psychosis. Biol Psychiatry. 2006;59(2):97-105.

25. Keefe RS, Sweeney JA, Gu H, et al. Effects of olanzapine, quetiapine, and risperidone on neurocognitive function in early psychosis: a randomized, double-blind 52-week comparison. Am J Psychiatry. 2007;164(7):1061-1071.

26. Olivier MR, Killian S, Chiliza B, et al. Cognitive performance during the first year of treatment in first-episode schizophrenia: a case-control study. Psychol Med. 2015;45(13):2873-2883.

27. Purdon SE, Jones BD, Stip E, et al. Neuropsychological change in early phase schizophrenia during 12 months of treatment with olanzapine, risperidone, or haloperidol. The Canadian Collaborative Group for research in schizophrenia. Arch Gen Psychiatry. 2000;57(3):249-258.

28. Csernansky JG, Mahmoud R, Brenner R, et al. A comparison of risperidone and haloperidol for the prevention of relapse in patients with schizophrenia. N Engl J Med. 2002;346(1):16-22.

29. Gaebel W, Schreiner A, Bergmans P, et al. Relapse prevention in schizophrenia and schizoaffective disorder with risperidone long-acting injectable vs quetiapine: results of a long-term, open-label, randomized clinical trial. Neuropsychopharmacology. 2010;35(12):2367-2377.

30. Kasper S, Lerman MN, McQuade RD, et al. Efficacy and safety of aripiprazole vs haloperidol for long-term maintenance treatment following acute relapse of schizophrenia. Int J Neuropsychopharmacol. 2003;6(4):325-337.

31. Pigott TA, Carson WH, Saha AR, et al. Aripiprazole for the prevention of relapse in stabilized patients with chronic schizophrenia: a placebocontrolled 26-week study. J Clin Psychiatry. 2003;64(9):1048-1056.

32. Ruhrmann S, Kissling W, Lesch OM, et al. Efficacy of flupentixol and risperidone in chronic schizophrenia with predominantly negative symptoms. Prog Neuropsychopharmacol Biol Psychiatry. 2007; 31(5):1012-1022.

33. Takeuchi H, Suzuki T, Remington G, et al. Effects of risperidone and olanzapine dose reduction on cognitive function in stable patients with schizophrenia: an open-label, randomized, controlled, pilot study. Schizophr Bull. 2013;39(5):993-998.

34. Bilder RM, Goldman RS, Volavka J, et al. Neurocognitive effects of clozapine, olanzapine, risperidone, and haloperidol in patients with chronic schizophrenia or schizoaffective disorder. Am J Psychiatry. 2002;159(6):1018-1028.
35. Roberts DL, Penn DL, Corrigan P, et al. Antipsychotic medication and social cue recognition in chronic schizophrenia. Psychiatry Res. 2010; $178(1): 46-50$

36. Lehman AF, Lieberman JA, Dixon LB, et al. Practice guideline for the treatment of patients with schizophrenia, second edition. Am J Psychiatry. 2004;161(2 suppl):1-56.

37. Gitlin M, Nuechterlein K, Subotnik KL, et al. Clinical outcome following neuroleptic discontinuation in patients with remitted recent-onset schizophrenia. Am J Psychiatry. 2001;158(11):1835-1842.

38. Tiihonen J, Haukka J, Taylor M, et al. A nationwide cohort study of oral and depot antipsychotics after first hospitalization for schizophrenia. Am J Psychiatry. 2011;168(6):603-609.

39. Bilder RM, Goldman RS, Robinson D, et al. Neuropsychology of firstepisode schizophrenia: initial characterization and clinical correlates. Am J Psychiatry. 2000;157(4):549-559.

40. Hoff AL, Svetina C, Shields G, et al. Ten year longitudinal study of neuropsychological functioning subsequent to a first episode of schizophrenia. Schizophr Res. 2005;78(1):27-34.

41. Wang CH, Li Y, Yang J, et al. A randomized controlled trial of olanzapine improving memory deficits in Han Chinese patients with firstepisode schizophrenia. Schizophr Res. 2013;144(1-3):129-135.

42. Schizophrenia Working Group of the Psychiatric Genomics C. Biological insights from 108 schizophrenia-associated genetic loci. Nature. 2014;511(7510):421-427.

43. Maier R, Moser G, Chen GB, et al. Joint analysis of psychiatric disorders increases accuracy of risk prediction for schizophrenia, bipolar disorder, and major depressive disorder. Am J Hum Genet. 2015; 96(2):283-294.

44. McFarlane WR, Cook WL, Downing D, et al. Portland identification and early referral: a community-based system for identifying and treating youths at high risk of psychosis. Psychiatr Serv. 2010;61(5): $512-515$.

45. Knowles EE, David AS, Reichenberg A. Processing speed deficits in schizophrenia: reexamining the evidence. Am J Psychiatry. 2010; 167(7):828-835.

46. Trusheim MR, Berndt ER, Douglas FL. Stratified medicine: strategic and economic implications of combining drugs and clinical biomarkers. Nat Rev Drug Discov. 2007;6(4):287-293.
Neuropsychiatric Disease and Treatment

\section{Publish your work in this journal}

Neuropsychiatric Disease and Treatment is an international, peerreviewed journal of clinical therapeutics and pharmacology focusing on concise rapid reporting of clinical or pre-clinical studies on a range of neuropsychiatric and neurological disorders. This journa is indexed on PubMed Central, the 'PsycINFO' database and CAS,

\section{Dovepress}

and is the official journal of The International Neuropsychiatric Association (INA). The manuscript management system is completely online and includes a very quick and fair peer-review system, which is all easy to use. Visit http://www.dovepress.com/testimonials.php to read real quotes from published authors. 